\title{
Fármacos y suplementos nutricionales para llevar en el botiquín del alpinista
}

\author{
Saioa Gómez-Zorita', Aritz Urdampilleta ${ }^{2,3,4}$ \\ 1. Departamento de Farmacia y Ciencias de los Alimentos. Facultad de Farmacia. Universidad del País Vasco (UPV/EHU). 2. Departamento \\ de Fisiología. Facultad de Farmacia. Universidad del País Vasco (UPV/EHU). 3. Centro Público para la Enseñanza de los Deportes, KIROLENE. \\ Gobierno Vasco. 4. Entrenamientos en Altitud e Hipoxia Intermitente. Unidad de Fisiología del Ejercicio. Centro Deportivo K2. Vitoria-Gasteiz. \\ NUTRIAKTIVE.
}

\section{PALABRAS CLAVE}

alpinismo, hipoxia,

fármacos, suplementos dietéticos, botiquín, mal agudo de montaña

\section{ABREVIATURAS}

AAS: ácido acetilsalicílico. MAM: mal agudo de montaña.

\section{KEYWORDS}

mountaineering, hypoxia, drugs, dietary supplements, first aid kit, acute mountain sickness

\section{RESUMEN}

Cada vez son más los individuos que acuden a montañas de gran altitud, con el riesgo que esta práctica entraña. Por tanto, es de gran importancia conocer los peligros y défıcits que esta práctica puede conllevar, con el fin de evitarlos o poderlos tratar. Es por ello que la preparación del botiquín, tanto con fines preventivos como paliativos o curativos tiene una gran transcendencia. Entre los suplementos nutricionales más importantes que debemos llevar están los multivitamínicos minerales, y entre los fármacos destacan aquellos para tratar y prevenir el mal agudo de montaña, destacando acetazolamida.

\section{Drugs and Dietary Supplements to be Carried in Mountaineers'}

First Aid Kits

\section{ABSTRACT}

There are a growing number of people who are climbing high mountains, with the risk that this sport involves. Therefore it is very important to be aware of the dangers and shortfalls that this practice can entail in order to prevent them and be able to treat them. Preparing one's first aid kit for preventative, palliative and therapeutic purposes is essential. We need to include multivitamin and mineral dietary supplements and drugs to treat and prevent acute mountain sickness, in particular Acetazolamide.
Financiación: Ninguna ajena.

Conflicto de intereses: Los autores declaran no existir conflicto de intereses en relación con el contenido del presente artículo. Cite este artículo como: Gómez-Zorita S, Urdampilleta A. Fármacos y suplementos nutricionales para llevar en el botiquin del alpinista. Farmacéuticos Comunitarios. 2014 Mar 01;6(1):29-34. doi:10.5672/FC.2173-9218.(2014/Vol6).001.06 Autora para correspondencia: Saioa Gómez-Zorita (saioa.gomez@ehu.es). 


\section{Introducción}

Cada vez es mayor el número de personas que acuden a hacer expediciones a altas montañas. Esto supone exponerse a la hipoxia hipobárica (reducción de la presión parcial de oxígeno) y a condiciones ambientales extremas, así como realizar actividad física extenuante, con el fin de alcanzar la cumbre, lo cual conlleva una serie de riesgos médicos y nutricionales por las condiciones anteriormente citadas, acompañados de una disminución de los recursos médicos y de los recursos nutricionales que se tienen al alcance (1).

Dadas las dificultades que entraña la ascensión a grandes altitudes, una buena planificación de los suplementos y fármacos a llevar es de vital importancia (2). Debemos conocer su utilidad y qué consecuencias puede acarrear su empleo.

A grandes altitudes (por encima de 4.000 metros), según la susceptibilidad individual a estados hipóxicos, es frecuente padecer el mal agudo de montaña (MAM) (3). Sus principales síntomas son cefalea, insomnio, anorexia y astenia (4), debido a una falta de adaptación a la altitud. Por otro lado, en casos más severos pueden aparecer alteraciones del estado mental, ataxia e, incluso, se puede llegar a dar la muerte del sujeto. Además de la susceptibi-

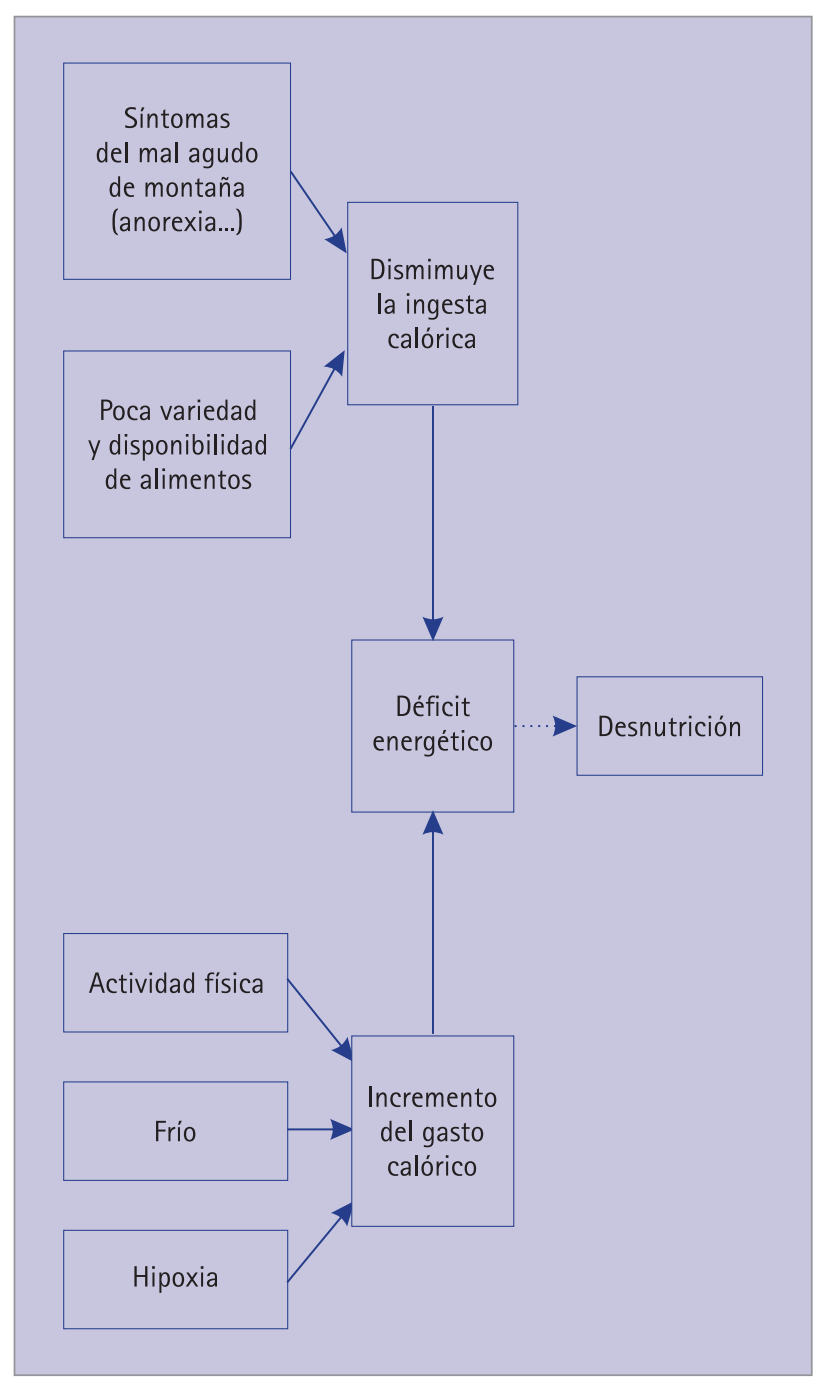

Figura 1 Consecuencia de las expediciones superiores a treinta dias en altura sobre el estado nutricional del alpinista (elaboración propia) lidad individual, parece ser que la mala forma física, mala alimentación-hidratación... pueden tener gran importancia en el MAM (5).

Otros problemas que pueden aparecer son traumatismos, infecciones, quemaduras y congelaciones, entre otros (6). Ante estas situaciones, para tratar estos problemas médicos, será necesario llevar la infraestructura necesaria desde el punto de vista farmacológico y del botiquín de primeros auxilios.

Aunque algunos autores han estudiado sobre los aspectos dieteticonutricionales en la alta montaña $(7,8)$, la importancia de la hidratación (2) o los riesgos mediconutricionales en el alpinismo (1) y los fármacos (acetozalamida) y suplementos (Ginkgo biloba) (9) necesarios para hacer frente al MAM, vemos importante hacer una tabla resumen de los fármacos que necesitaría llevar el alpinista a sus expediciones y, desde el punto de vista farmacológico, explicar por qué podría ser de utilidad en la montaña, así como dar indicaciones de la dosis y otros aspectos de interés.

Así, es importante saber cómo actuar ante situaciones o enfermedades que pueden aparecer en montañas de gran altitud cuando no haya un profesional sanitario a quien consultar (8). Por otro lado, el farmacéutico comunitario puede colaborar con el deportista en la elaboración de un botiquín de primeros auxilios adecuado a sus necesidades específicas.

\section{Método}

Se ha realizado una búsqueda bibliográfica sobre los fármacos utilizados en el alpinismo, tanto para prevenir el MAM como posibles problemas debido a una mala respuesta a la altitud $u$ otros que puedan surgir en la montaña. Para ello, se han utilizado los buscadores como: PUBMED (MedLine), SPORDISCUS, Scielo y Google Académico como motor de búsqueda y, a su vez, se consultó en el vademécum sobre los fármacos (www. vademecum.es).

Por otra parte, se realizó la estrategia de bola de nieve para intentar buscar más artículos de interés en la temática. No se han acotado los años de búsqueda y las palabras clave utilizadas fueron: alpinismo, montañismo, fármacos, suplementos, vitaminas, minerales, mal agudo de montaña, hipoxia.

\section{Resultados}

Las expediciones a altas montañas entrañan una serie de riesgos que ponen en peligro la vida del alpinista, por lo que es de vital importancia cuidar hasta el último detalle durante las expediciones (10). Además, si se quiere realizar cumbre, se debe estar en las condiciones más óptimas que sean posibles.

\section{Suplementos nutricionales}

En primer lugar, se puede observar cómo en las estancias a elevadas altitudes, puede haber cierto grado de desnutrición (figura 1) (11). Por tanto, se debería prestar atención a la ingesta de alimentos y, si fuese necesario utilizar, suplementos, tal y como indicaremos a continuación. 
Además, la hipoxia, per se, incrementa las pérdidas de agua, tanto por vía renal como pulmonar (figura 2), lo que conlleva un mayor riesgo de deshidratación (12).

El balance hídrico es esencial para el adecuado funcionamiento de los órganos. El agua es el medio donde ocurren las reacciones del organismo, y es esencial para mantener el volumen sanguíneo y, de este modo, la integridad del sistema cardiovascular. Cada compartimento acuoso tiene una concentración dada de electrolitos que regula el volumen de agua de cada uno de los compartimentos y mantiene el potencial electroquímico de la membrana (13). En el líquido extracelular, el principal catión es el sodio $(\mathrm{Na})$ y el principal anión, el cloro $(\mathrm{Cl})$. En cambio, el potasio $(\mathrm{K})$ es el principal catión intracelular. Los tres son esenciales para mantener el balance de fluidos y electrolitos.

Durante la actividad física hay gran pérdida no solo de agua, sino también de electrolitos, por el sudor. Por tanto, dado el peligro que ello puede conllevar, se debería ingerir agua con electrolitos, es decir, ligeramente hipertónica. El déficit de $\mathrm{Na}$ y/o una excesiva ingesta de líquido sin sales $(\mathrm{Na}, \mathrm{K} \mathrm{y} \mathrm{Cl})$ pueden ser un factor desencadenante de hiponatremia.

Las altas ingestas de agua que se requieren en el alpinismo hacen que sean un colectivo de riesgo de padecer hiponatremia, siempre y cuando no se aporten sales. Como norma general se requiere $0,5-0,7 \mathrm{~g} \mathrm{Na} / \mathrm{L}$ de líquido ingerido, es decir, $2 \mathrm{~g}$ de sal común (1), ya que la sal común contiene cloro y sodio, en un porcentaje 70/40\%, respectivamente.

Respecto a las carencias nutricionales, podemos encontrar déficit de vitaminas hidrosolubles (necesitan ser ingeridas a diario, ya que no se almacenan en el organismo) y de minerales importantes como el calcio (Ca), magnesio ( $\mathrm{Mg}$ ) o hierro (Fe), debido a la escasa diversidad de alimentos que son ingeridos (1). Por esta razón podrían ser administrados multivitamínicos y multiminerales. Como norma general, parece una estrategia interesante, antes de acudir a las montañas elevadas, cuando se prevén estancias superiores a las tres semanas, hacer una "carga" de vitaminas liposolubles, y así no es necesaria la toma de estas en la propia montaña, ya que al ser liposolubles (vitamina A, D o E) se almacenan en el tejido adiposo del alpinista-turista (1). Además, se ha visto que la vitamina E (antioxidante) puede ayudar a contrarrestar el elevado estrés oxidativo que supone esta práctica $(2,14)$.

El déficit de Fe es uno de los riegos nutricionales que se tienen que suplir en las estancias superiores a 2-3 semanas, porque la dieta del alpinista es deficitaria en energía y proteínas de origen animal, tanto por déficit en la ingesta como por un incremento en su excreción. Por tanto, podría estar aconsejada la suplementación de Fe (200$300 \mathrm{mg} /$ día), preferiblemente acompañado de vitamina C ( $1 \mathrm{~g} /$ día) para favorecer su absorción. Esta ingesta del Fe debería ser en ayunas $(1,15)$.

El Ca es un mineral de gran importancia, cuyo déficit de forma puntual y aguda puede ocasionar alteraciones de la contracción muscular y calambres musculares, aunque generalmente en estos casos suele ser extraído el Ca de los huesos para evitar una disminución de su concentración en sangre (11). Observar los efectos del déficit de $\mathrm{Mg}$ no es habitual, siendo sus síntomas calambres musculares y debilidad muscular principalmente.

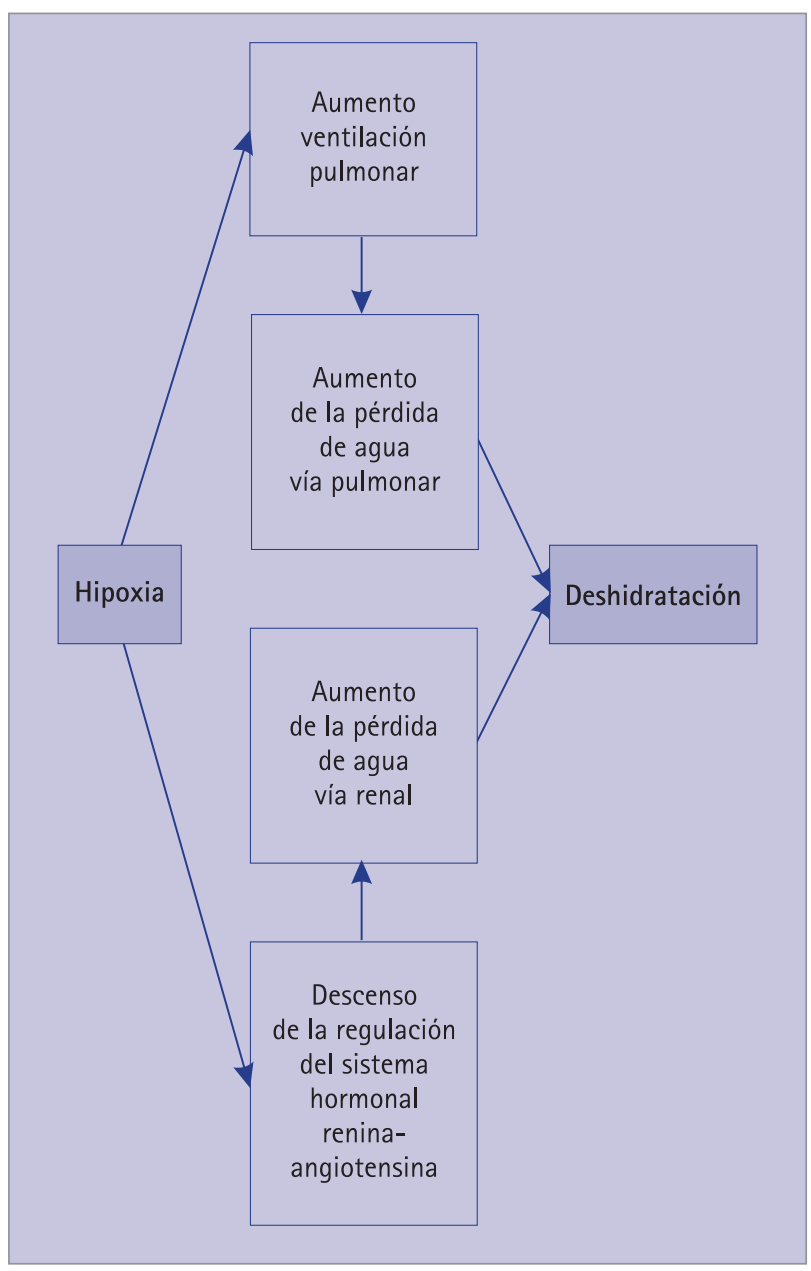

Figura 2 Efecto de la hipoxia sobre la hidratación (elaboración propia)

Otros problemas observados son las alteraciones inmunológicas que se dan durante las expediciones a altas montañas (16), por lo que se podrían utilizar probióticos, glutamina o incluso AM3 (glucofosfopeptical) con el fin de prevenir estas alteraciones inmunológicas y con fines inmunomoduladores.

Por otro lado, el Ginkgo biloba ha sido ampliamente estudiado como agente para prevenir y tratar el MAM, así como el edema cerebral. Sin embargo, los resultados obtenidos han sido muy dispares, por lo que no se recomienda su utilización en el alpinismo, ni como medida preventiva ni paliativa, ya que los estudios realizados no muestran una eficacia segura y es necesario realizar más estudios (17).

\section{Medicamentos}

En cuanto a los medicamentos, conviene saber que una buena planificación de los medicamentos que se deben llevar a las expediciones es de vital importancia. Si tenemos en cuenta que por lo general las heridas, golpes, accidentes y patologías que ocurren en la montaña no se pueden tratar de inmediato, disponer de algunos productos y materiales con los que atender el problema de forma rápida puede llevar incluso a salvarnos la vida. Por otro lado, como se ha dicho anteriormente, es posible padecer MAM, patología a tener en cuenta a la hora de preparar el botiquín (18). 
Tabla 1 Propuesta de medicamentos a llevar a expediciones a montañas de gran altitud

\begin{tabular}{|c|c|c|c|c|c|}
\hline Principio activo & Dosis & Efecto & Empleo & Dosificación & Otras observaciones \\
\hline $\begin{array}{l}\text { Ácido } \\
\text { acetilsalicílico } \\
\text { (AAS) }\end{array}$ & $300 \mathrm{mg}$ & $\begin{array}{l}\text { Antiagregante } \\
\text { plaquetario }\end{array}$ & Congelaciones & $300 \mathrm{mg} / 24 \mathrm{~h}$ & $\begin{array}{l}\text { No con el estómago vacío } \\
\text { Comprimido }\end{array}$ \\
\hline Enoxaparina & $20 \mathrm{mg} / 0,2 \mathrm{ml}$ & Anticoagulante & Congelaciones & $20 \mathrm{mg} / 24 \mathrm{~h}$ & $\begin{array}{l}\text { Inyectable } \\
\text { Junto con AAS } \\
\text { No utilizar con acetaminofén (pa- } \\
\text { racetamol) y en la medida de lo } \\
\text { posible tampoco con diuréticos }\end{array}$ \\
\hline Acetazolamida & $250-500 \mathrm{mg}$ & Diurético & $\begin{array}{l}\text { Prevención y trata- } \\
\text { miento del mal agudo } \\
\text { de montaña (MAM) }\end{array}$ & $\begin{array}{l}250 \mathrm{mg} / 12 \mathrm{~h} \\
500 \mathrm{mg} \text { si liberación } \\
\text { sostenida/24 h }\end{array}$ & $\begin{array}{l}\text { Si es de liberación sostenida no } \\
\text { masticar ni romper } \\
\text { Evitar AAS }\end{array}$ \\
\hline Ibuprofeno & 400 o $600 \mathrm{mg}$ & $\begin{array}{l}\text { Analgésico y } \\
\text { antiinflamatorio }\end{array}$ & Dolor o inflamación & $400-600 \mathrm{mg} / 8 \mathrm{~h}$ & $\begin{array}{l}\text { Preferiblemente junto con ali- } \\
\text { mentos } \\
\text { Comprimido o sobres }\end{array}$ \\
\hline Metamizol & $575 \mathrm{mg}$ & $\begin{array}{l}\text { Analgésico } \\
\text { Antipirético }\end{array}$ & $\begin{array}{l}\text { Dolor agudo si el } \\
\text { analgésico de prime- } \\
\text { ra línea no es sufi- } \\
\text { ciente }\end{array}$ & $575 \mathrm{mg} / 8 \mathrm{~h}$ & $\begin{array}{l}\text { Se puede combinar con ibuprofeno } \\
\text { dejando un intervalo de tiempo } \\
\text { entre ambos de } 4 \mathrm{~h} \\
\text { Cápsulas } \\
\text { No masticar las cápsulas ni abrir }\end{array}$ \\
\hline Diclofenaco gel & & $\begin{array}{l}\text { Antiinflamatorio } \\
\text { tópico }\end{array}$ & $\begin{array}{l}\text { Contusión sin } \\
\text { herida }\end{array}$ & 1 aplicación/8 h & Gel \\
\hline Loperamida & $2 \mathrm{mg}$ & Antidiarreico & Diarrea & $\begin{array}{l}\text { Cada vez que haya } \\
\text { deposición, se puede } \\
\text { empezar tomando } 2 \\
\text { a la vez (máximo } 6 \\
\text { cápsulas/dia) }\end{array}$ & $\begin{array}{l}\text { Si más de } 3 \text { dias seguidos parar } \\
\text { tratamiento y lo mismo si hay } \\
\text { fiebre (posible infección) }\end{array}$ \\
\hline Metoclopramida & $10 \mathrm{mg}$ & Antiemético & Náuseas y vómitos & $10 \mathrm{mg} / 6 \mathrm{~h}$ & $\begin{array}{l}\text { Evitar paracetamol y aspirina si es } \\
\text { posible } \\
\text { Cápsulas (no romper ni masticar) }\end{array}$ \\
\hline $\begin{array}{l}\text { Amoxicilina/ } \\
\text { ácido } \\
\text { clavulánico }\end{array}$ & $875 \mathrm{mg} / 125 \mathrm{mg}$ & Antibiótico & $\begin{array}{l}\text { Heridas sin asisten- } \\
\text { cia médica antes de } \\
6 \text { h Infección vias } \\
\text { respiratorias y uri- } \\
\text { narias }\end{array}$ & Cada 8 h/7-10 días & $\begin{array}{l}\text { Ciprofloxacino ( } 500 \mathrm{mg} \text { ) en caso de } \\
\text { alergia } \\
\text { Evitar su utilización con anticoa- } \\
\text { gulantes si es posible } \\
\text { No ingerir con antiácidos }\end{array}$ \\
\hline Mupirocina & $20 \mathrm{mg} / \mathrm{g}$ & $\begin{array}{l}\text { Antibiótico } \\
\text { tópico }\end{array}$ & Infección piel & Cada 8-12 h & $\begin{array}{l}\text { Pomada } \\
\text { Se puede cubrir la zona con gasas }\end{array}$ \\
\hline Temazepam & $10 \mathrm{mg}$ & Sedante & Insomnio & $1 \mathrm{mg} / \mathrm{dia}$ & $\begin{array}{l}\text { En la cena } \\
\text { Si es posible, evitar su utilización }\end{array}$ \\
\hline Omeprazol & $20 \mathrm{mg}$ & $\begin{array}{l}\text { Inhibidor bomba } \\
\text { protones }\end{array}$ & $\begin{array}{l}\text { Acidez, ardor esto- } \\
\text { macal }\end{array}$ & $20 \mathrm{mg} / 24 \mathrm{~h}$ & \\
\hline Cetirizina & $10 \mathrm{mg}$ & Antihistamínico & Alergia & $10 \mathrm{mg} / 24 \mathrm{~h}$ & $\begin{array}{l}\text { Comprimidos recubiertos (no mas- } \\
\text { ticar ni romper) }\end{array}$ \\
\hline
\end{tabular}

Así, en la tabla 1 podemos observar lo que sería un botiquín básico para llevar expediciones a montañas de gran altitud, en donde la patología principal es el MAM y los accidentes pueden ser dramáticos.

Para prevenir y tratar el MAM, el medicamento más empleado y más útil es acetazolamida, el medicamento de primera línea para esta patología (18). Acetazolamida es un diurético inhibidor de la anhidrasa carbónica renal que incrementa la pérdida de bicarbonato, sodio y potasio por vía renal. Esto da lugar a una mayor hiperventilación con el fin de evitar la acidosis metabólica. Este fármaco se utiliza tanto para la prevención como el tratamiento del MAM (125-250 mg/8-12 h). En el caso de emplearse como agente preventivo, se debería comenzar su administración un día antes de comenzar el ascenso y hasta 2-5 días después de alcanzar la altitud (19). Las reacciones 
adversas que ocasiona son poco frecuentes y destacan las parestesias, alteraciones gastrointestinales y somnolencia.

En casos extremos del MAM, es frecuente recurrir a la utilización de oxígeno o cámaras hiperbáricas (20). Dexametasona también puede ser efectiva para prevenir y tratar el MAM. No obstante, no ayuda a la aclimatación del montañero (4). Por tanto, sería un fármaco de segunda línea en la prevención del MAM, pero sí que se ha visto que es de gran utilidad cuando se padece un edema cerebral y pulmonar $(17,21)$.

En el tratamiento de la cefalea causada por el MAM se pueden emplear diversos analgésicos como AAS (300 mg/ 4-6 h), ibuprofeno (600 mg/8 h), naproxeno (550 mg/8 h) o paracetamol ( $1 \mathrm{~g} / 8 \mathrm{~h}$ ) (21). En caso de úlcera, se recomendaría la utilización de paracetamol (no antiinflamatorio) frente a ibuprofeno y AAS. Si hay dolores severos (traumatismos...) se podría combinar paracetamol con otro analgésico como ibuprofeno, combinándolos y separando su toma 4 horas. En caso de que no hagan efecto se podría utilizar metamizol. Estos fármacos, al suprimir las molestias (enmascaramiento), pueden inducir a continuar con la ascensión y, por tanto, pueden exponer al individuo a padecer formas más graves de MAM. Los principales efectos secundarios que producen son a nivel gastrointestinal.

Para tratar el insomnio tradicionalmente se utilizan benzodiazepinas como lorazepam o diazepam (21). Sin embargo, la mayoría de ellas causan depresión respiratoria, por lo que su uso no estaría recomendado. En caso de utilizarse, se ha observado que temazepam (10 mg/noche) podría ser el más indicado (18), dado que no causa depresión respiratoria.

En caso de padecer edema cerebral, el tratamiento de primera elección consiste en la administración de corticoides como dexametasona (8 $\mathrm{mg}$ dosis inicial y posteriormente $4 \mathrm{mg} / 6 \mathrm{~h}$ ) o prednisona (50-100 $\mathrm{mg}$ y posteriormente $50 \mathrm{mg} / 8-12 \mathrm{~h}$ ) hasta descender o hasta que desaparezcan los síntomas. Se ha visto que dexametasona también puede ser administrada como medida profiláctica para el edema cerebral $(4 \mathrm{mg} / 6 \mathrm{~h})$.

En caso de edema pulmonar, se utiliza tanto para la prevención como para el tratamiento nifedipina (10 mg/ $15 \mathrm{~min} 2$ veces y posteriormente $20 \mathrm{mg} / 8-12 \mathrm{~h}$ ), cuyo mecanismo de acción consiste en el bloqueo de los canales de calcio. También se puede emplear salmeterol (125 pg/12 h), que facilita la difusión alveolar, e inhibidores de la fosfodiesterasa como sildenafilo $(20 \mathrm{mg} / 6-8 \mathrm{~h})$ y tadalafilo $(7,5-$ $10 \mathrm{mg} / 12 \mathrm{~h}$ ) (17), ya que reducen la hipertensión pulmonar. A su vez, dexametasona suele resultar ineficaz en el caso del edema pulmonar, especialmente en sujetos parcialmente aclimatados o cuando tienen una historia previa de dicha enfermedad, por lo que en este caso su utilización no estaría justificada.

En cuanto a la administración de antibióticos (principalmente amoxicilina o ciprofloxacino), únicamente se emplearán si se sospecha infección, como puede ser en el caso de padecer neumonía (21).

En el caso de edemas periféricos, se pueden emplear diuréticos si, descendiendo de altitud, no se llegan a controlar (17), aunque raramente es necesario. En este caso, deberíamos controlar la pérdida de líquidos y mantenernos bien hidratados.

Asimismo, en caso de padecer congelaciones, se recomienda la utilización de ácido acetilsalicílico para mejorar la circulación y mitigar el dolor, junto con una heparina de bajo peso molecular como enoxaparina (22). De nuevo en este caso los efectos secundarios que puedan derivar de este tratamiento pueden ser un mal menor dado el impacto que tienen las congelaciones en el individuo. No obstante, no cabe duda que ante las congelaciones la mejor opción es su prevención. Una correcta hidratación en la montaña con bebidas isotónicas será lo más seguro, ya que en un estado de deshidratación elevada a altas altitudes el organismo, para proteger los órganos vitales, ejerce una vasodilatación periférica y, en consecuencia, una mayor susceptibilidad a las congelaciones.

Finalmente, se deben llevar otros medicamentos para utilizar en caso de sufrir algún otro tipo de incidentes como contusiones, pequeños traumatismos o algunas otras alteraciones como la diarrea. Así, por ejemplo, para el tratamiento de la diarrea se recomienda la utilización de loperamida, siempre y cuando no se sospeche de infección.

\section{Conclusión}

Como se ha observado, no es fácil la preparación del botiquín del alpinista, y un gran número de consideraciones han de tenerse en cuenta a la hora de prepararlo. Asimismo, se debería disponer de todo aquello que pudiéramos necesitar hasta ponernos en manos de un profesional sanitario, e intentar que nuestro organismo esté en condiciones óptimas para soportar las posibles desavenencias a las que la expedición nos pueda conllevar.

Por otro lado, cabe recordar que es primordial mantener un adecuado balance hídrico, por lo que deberíamos aportar agua y sales minerales al organismo y evitar ciertas carencias nutricionales, con lo que se deberían llevar a la expedición vitaminas hidrosolubles y algunos minerales como el hierro. Finalmente, entre los medicamentos que no deberíamos olvidar están la acetazolamida, analgésicos, corticoides y antibióticos.

\section{Referencias bibliográficas}

1. Urdampilleta A, Martínez-Sanz JM. Riesgos médico-nutricionales y planificación dietética en el alpinismo. Motricidad. European Journal of Human Movement. 2012;28:3566.

2. Westerterp KR. Energy and water balance at high altitude. News in Physiolical Sicences. 2011;16:134-7.

3. Dumont L, Mardirosoff C, Tramer MR. Efficacy and harm of pharmacological prevention of acute mountain sickness: quantitative systematic review. British Medical Journal. 2000;321:267-72. doi:10.1136/bmj.321.7256.267

4. Hackett PH, Roach RC. High-altitude illness. The New England Journal of Medicine. 2001;345(2):107-14. doi:10.1056/NEJM 200107123450206

5. Wagner DR, Fargo, JD, Parker D, Tatsu-gawa K, Young TA. Variables contributing to acute mountain sickness on the summit of Mt Whitney. Wilderness \&t Environmental Medicine. 2006;17:221-8. doi:10.1580/PR43-05.1

6. Martinez-Carpio PA, Battestini R. Medicina de montaña en el año 2002 (Año Internacional de las Montañas de las Naciones Unidas). Pasado, presente y futuro. Med Clin (Barc). 2002;119(20):776-84. doi:10.1016/S0025-7753(02)73577-X

7. Oliver SJ, Golja P, Macdonald JH. Carbohydrate supplementation and exercise performance at high altitude: a randomi-zed controlled trial. High Alt Med Biol. 2012;13(1):22-31. doi:10.1089/ham.2011.1087 
8. Kechijan D. Optimizing nutrition for performance at altitude: a literature review. Journal of Special Operartions Medicine: a peer reviewed. 2011;11:12-7.

9. Chow T, Browne V, Heileson HL, Wallace D, Anholm J, Green SM. Ginkgo biloba and acetazola mide prophyla xis for acute mountain sickness: a randomized, place-bo-controlled trial. Archive of Internal Medicine. 2005;165:296-301. doi:10.1001/archinte.165.3.296

10. Westhoff JL, Koepsell TD, Littell CT. Effects of experience and commerciali-sation on survival in Himalayan mountaineering: retrospective cohort study. BMJ. 2012;344:e3782. doi:10.1136/bmj.e3782

11. Mayor F, Cascales M. Enfermedades metabólicas. Monografías XX, Real Academia Nacional de Farmacia. 2006.

12. Yanagisawa K, Ito O, Nagai S, Onishi S. Electrolyte-carbohydrate beverage prevents water loss in the early stage of high altitude training. The Journal of Medical Investigation. 2012;59:102-10. doi:10.2152/jmi.59.102

13. Sawka MN, Mountain SJ. Fluid and electrolyte supplementation for exercise heat stress. Am J Clin Nutr. 2000;72(2):56472.

14. Warner PD. Operation Everest II. High Altitude Medicine \&t Biology. 2010;11(2):111-9. doi:10.1089/ham.2009.1084

15. Mariscal-Arcas M, Carvajal C, Monteagudo C, Lahtinen J, Fernandez de Alba C, Feriche B et al. Nutritional analysis of diet at base camp of a seven thousandmeter mountain in the Himalayas. Revista Andaluza de Medicina del Deporte. 2010;3(4):127-32.

16. Mishra KP, Ganju L. Influence of High Altitude Exposure on the Immune System: A Review. Immunological Investigations. 2010;39(3):219-34. doi:10.3109/08820131003681144

17. Fiore DC, Hall S, Shoja P. Altitude Illness: Risk Factors, Prevention, Presentation, and Treatment. American Family Physician. 2010;82(9):1103-10.

18. Luks AM. Which medications are safe and effective for improving sleep at high altitude?. High Alt Med Biol. 2008;9:195-8. doi:10.1089/ham.2008.1025

19. Low EV, Avery AJ, Gupta V, Schedlbauer A, Grocott MP. Identifying the lowest effective dose of acetazolamide for the prophylaxis of acute mountain sickness: systematic review and metaanalysis. BMJ. 2012;345:e6779. doi:10.1136/ bmj.e6779

20. Netzer N, Strohl K, Faulhaber M, Gatterer H, Burtscher M. Hypoxia-Related Altitude Illnesses. Journal of Travel Medicine. 2013;20(4):247-55. http://dx.doi.org/10.1111/jtm. 12017

21. Strapazzon G, Procter E, Brugger H. The quest for evidence-based medicine in mountain areas. High Alt Med Biol. 2011;12(4):399-400. doi:10.1089/ham.2011.1058

22. García R, Soto JM, Barranco F. Patología derivada de la altura: experiencia en el K2. Medicina Intensiva. 2006;30(5):2325. doi:10.1016/S0210-5691(06)74512-0 